Measur ement of net group and reshaping del ays for opt i cal pul ses in di sper si ve nedi a

\begin{tabular}{|l|l|}
\hline 著者 & $\begin{array}{l}\text { Tal ukder Ami nul, Har ut a Takat oshi, Tom ta } \\
\text { Nakot o }\end{array}$ \\
\hline $\begin{array}{l}\text { j our nal or } \\
\text { publ i cat i on t i t l e }\end{array}$ & Physi cal Revi ew Let ter s \\
\hline vol une & 94 \\
\hline number & 22 \\
\hline page range & $223901-223901$ \\
\hline year & 2005- 06 \\
\hline 出版者 & Aner i can Physi cal Soci et y \\
\hline 権利 & (c) 2005 The Aner i can Physi cal Soci et y \\
\hline URL & ht t p: //hdl . handl e. net /10297/583 \\
\hline
\end{tabular}




\title{
Measurement of Net Group and Reshaping Delays for Optical Pulses in Dispersive Media
}

\author{
Aminul I. Talukder, Takatoshi Haruta, and Makoto Tomita \\ Department of Physics, Faculty of Science, Shizuoka University, 836 Ohya, Suruga-ku, Shizuoka 422-8529, Japan
}

(Received 7 December 2004; published 9 June 2005)

\begin{abstract}
We experimentally demonstrate the direct measurement of net group and reshaping delays for arbitrary optical pulses in dispersive media, verifying the earlier prediction of Peatross et al. [Phys. Rev. Lett. 84, 2370 (2000)]. Incoherent pulse propagation in an absorptionless system is well described by net group delay; even the medium causes a great deal of deformation in the transmitted pulse. However, in the case of phase modulated chirping pulses in a resonant absorber, the so-called superluminal or subluminal propagation velocity is strongly influenced by the reshaping delay.
\end{abstract}

DOI: $10.1103 /$ PhysRevLett.94.223901

PACS numbers: $42.25 . \mathrm{Bs}$

The velocity of the wave packet, which may be highly subluminal, superluminal, or even negative, has a great significance in physics. Historically, the study of wave packet propagation in dispersive media was started by Sommerfeld and Brillouin [1]. Garret and McCumber [2] predicted that "the pulse peak through a small optical thickness of a resonant absorber can propagate with the conventional group velocity even it is superluminal or negative." Superluminal pulse propagation has been experimentally observed through a resonant absorber [3], single photon tunneling [4], birefringent photonic crystals, etc. [5-7]. Another current interest, in pulse propagation, may be the ultraslow group velocity observed in atomic gases [8-10]. The key point is that the conventional group velocity can explain pulse propagation for limited cases, that is, for small optical thickness of the medium, narrow input, and/or broad absorption line.

Superluminal group velocity, especially in the context of describing the signal or information propagation, has renewed the fundamental interest [11]. Such velocity appears in the anomalous dispersion region with severe attenuation or amplification. So, the identity of the transmitted pulse with the incident one as well as the concept of superluminal signal transfer becomes dubious. To overcome this situation, Wang et al. created a transparent anomalous dispersion region between two amplification lines and observed the superluminal propagation without a significant attenuation, amplification, or deformation in the pulse shape [12]. Their achievement is a major breakthrough in superluminal phenomena. In physical systems, however, as well as in applications, lossless and group velocity dispersion free region is a very special case because such a system may hardly appear even in a well designed and careful experimental technique. Furthermore, as for signal, if one wishes to encode a nonanalytical structure on the pulse shape, the pulse spectrum becomes nearly infinite in width and the narrow band treatment of the pulse naturally breaks down. In this respect, an important question in physics is whether it is possible to describe the pulse propagation beyond the conventional group velocity, espe- cially where a strong pulse attenuation, amplification, or distortion occurs.

Peatross et al. [13] have defined the arrival time of a pulse by the expectation integral, $\langle t\rangle_{\vec{r}}=\int t S(\vec{r}, t) d t /$ $\int S(\vec{r}, t) d t$, where $\vec{S}(\vec{r}, t)$ is the Poynting vector in the pulse. In their analysis, it is asserted that the propagation velocity, in terms of net group and reshaping delays, is always significant even in the cases of strong attenuation, amplification, or distortion. In this Letter, we report experimental investigation of the propagation of arbitrarily shaped femtosecond optical pulses through various dispersive media and thus the measurement of propagation delay. Definitely the new concept of group velocity does not break down in any case.

The experimental setup consists of a dual interferometer, and is shown in Fig. 1. Transform limited femtosecond optical pulses, with a temporal width of $70 \mathrm{fs}$, from a Ti:sapphire laser, are used. A double-pulsed signal is obtained with the first (Mach-Zehnder) interferometer in which the reference arm is empty and the sample arm contains the sample. The difference in the optical path lengths $\Delta L$ of the two arms of the interferometer is scanned by corner cube reflector $\mathrm{CC} 1$, on a translational stage in the reference arm, and measured by a precise magnetic position sensor. To have arbitrary pulses in the

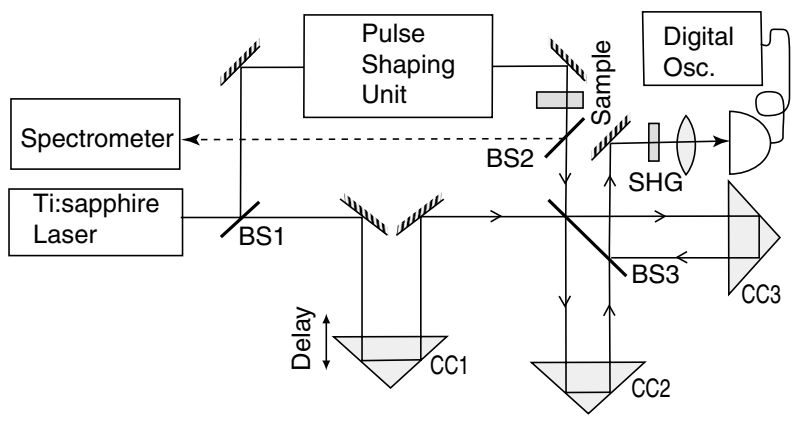

FIG. 1. Schematic experimental setup: Beam splitters; BS1, $\mathrm{BS} 2$, and BS3. Corner cube reflectors: $\mathrm{CC} 1, \mathrm{CC} 2$, and $\mathrm{CC} 3$; second harmonic generating crystal, SHG. 
sample arm, the sample beam was passed through a pulse shaping unit where the incoming pulses could be modified and shaped into complex pulses with multiple peaks or into chirping pulses. In order to form pulses with multiple peaks, the pulse shaping apparatus was configured in a 4-F arrangement [14]. A pair of gratings (1200 line/mm), placed at the focal planes of a unit magnification confocal lens pair with focal lengths of $\mathrm{F}=14 \mathrm{~cm}$, was the main element in the pulse shaping unit. Spatially patterned phase and amplitude masks, fabricated on a half of a glass substrate, were inserted between the lenses by mounting on a translation stage. The masks could be slid into a position that the beam could either pass or avoid to pass through the masks, and thus the complex pulses with multiple peaks or incoming coherent pulses were obtained. Nevertheless, the beam always took the same glass substrate; the setup could determine the relative temporal position of both pulses with an accuracy of \pm 1 fs. An example of produced complex pulses is shown in Fig. 2(a). We describe such nontransform limited multipeaked pulses as incoherent pulses in this Letter. To form chirping pulses, the gratings pair were simply placed with a separation of about $8.1 \mathrm{~mm}$ between them. The sample and reference beams emerging from the first interferometer were carefully combined by the beam splitter BS3 and led into the second (Michelson) interferometer. Thus, each arm of the second interferometer contained a pair of pulses: an undisturbed reference pulse and an incoherent, or a chirping, or a coherent pulse, transmitted by the sample. Therefore, the intensity correlation signal of the pulse pair is obtained by scanning the time delay $\tau$ between two copies of the pair. Near $\tau=0$ the sample pulse overlaps with its own copy and so does the reference pulse. The measured signal is then the sum of the intensity autocorrelations of the two pulses. Thus, for every scan, the center of mass of autocorrelation gives the starting point $(\tau=0)$. Near $\tau= \pm \Delta L / c$, with $c$ the speed of light, one copy of the sample pulse overlaps with one copy of the reference pulse. This produces the cross correlation trace of the sample and reference pulses. The correlation trace is averaged and then the propagation delay, that is, the temporal separation between the centers of mass of autocorrelation and cross correlation, has been determined using a computational data processing routine. So, our double interferometer setup has merit: determining the propagation delay of arbitrarily shaped pulses with a good accuracy using the autocorrelation as a standard. The spectrum of the transmitted pulse also is fed into a $25 \mathrm{~cm}$ spectrometer and recorded using a charge-coupled device camera.

The concept of both net group and reshaping delays may be examined individually in a well designed experimental system. We first examine the propagation of both coherent and incoherent optical pulses in order to verify the significance of net group delay. In this case, an absorptionless dispersive medium, $\mathrm{ZnSe}$ substrates, is used as the sample and inserted into the sample arm of the first interferometer as shown in Fig. 1. The refractive index

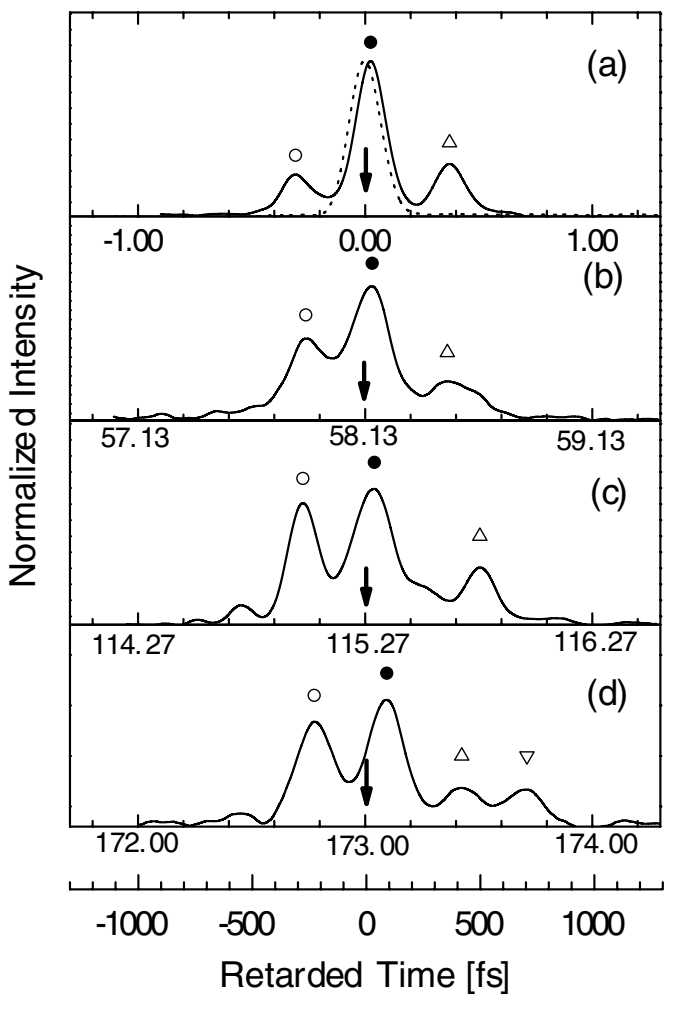

FIG. 2. Observed cross correlations (solid curves) of incoherent pulses, at $800 \mathrm{~nm}$, transmitted through $\mathrm{ZnSe}$ of (a) 0.0 , (b) 10.10 , (c) 20.03 , and (d) $30.07 \mathrm{~mm}$, in the sample arm. The cross correlation represented by the dashed line in (a) is for coherent pulses at the same wavelength. The horizontal axis for each graph is the absolute delay time $\tau_{\text {abs }}$ and labeled in ps. The centers of mass for coherent pulses transmitted through different lengths of $\mathrm{ZnSe}$ are (a) 0.0, (b) 58.13, (c) 115.27, and (d) $173.00 \mathrm{ps}$, while the corresponding ones for incoherent pulses are indicated by downward arrows. Open circles, solid circles, and upward triangles are the eye guides to three major peaks of cross correlations. The cross correlation is seen to widen and deformed significantly, increasing ZnSe thickness, evolving an additional peak shown by the downward triangle in (d).

curve of $\mathrm{ZnSe}$ is given by an approximation equation, $n=$ $\sqrt{\left[3.882+2.066 \lambda^{2} /\left(\lambda^{2}-0.109\right)\right]}$, with $\lambda$ in $\mu \mathrm{m}$. Figure 2 shows the cross correlations for incoherent pulses after propagation through different lengths of ZnSe. The horizontal axis for each graph is labeled in ps and describes the absolute delay time $\tau_{\mathrm{abs}}$ in which the origin, $\tau_{\mathrm{abs}}=0$, is taken at the center of mass of the coherent pulse without the $\mathrm{ZnSe}$ sample. The additional axis in the bottom displays the retarded delay time $\tau_{\text {ret }}$ in which the origin, $\tau_{\text {ret }}=$ 0 , is taken at the center of mass of the coherent pulse with corresponding length of $\mathrm{ZnSe}$. The absolute propagation time, determined at the center of mass, of transmitted incoherent pulse coincides with that of the transmitted coherent pulse with an accuracy of \pm 2 fs, as indicated by the downward arrows in all experiments [graphs (a) to (d)]. This is true even for a long propagation distance $(\cong 200 \mathrm{ps})$ with a severe distortion in the pulse shape as shown in 
Fig. 2(d). It would be worthwhile to note that no spectral shifts were observed in the transmitted pulse even for a $40 \mathrm{~mm}$ thickness of $\mathrm{ZnSe}$.

It is clear that the deformation in the transmitted pulse profile may occur when it travels a long propagation distance through a dispersive medium and thus the conventional group velocity loses precise meaning. In order to describe this situation, it has been suggested [13] that if one describes the propagation time by the arrival of the center of mass of the pulse, then the time interval between the pulse's arrival at two points through the medium is given by

$$
\begin{aligned}
\Delta t= & \frac{\hat{u} \cdot \int_{-\infty}^{\infty} \vec{S}(\vec{r}, \omega)\left[\frac{\partial \operatorname{Re} \vec{k}}{\partial \omega} \cdot \Delta \vec{r}\right] d \omega}{\hat{u} \cdot \int_{-\infty}^{\infty} \vec{S}(\vec{r}, \omega) d \omega}+T\left[e^{-\operatorname{Im} \vec{k} \cdot \Delta \vec{r}} \vec{E}\left(\vec{r}_{0}, \omega\right)\right] \\
& -T\left[\vec{E}\left(\vec{r}_{0}, \omega\right)\right],
\end{aligned}
$$

where

$$
T[\vec{E}(\vec{r}, \omega)]=-i \frac{\hat{u} \cdot \int_{-\infty}^{\infty} \frac{\partial \vec{E}(\vec{r}, \omega)}{\partial \omega} \times \vec{H}^{*}(\vec{r}, \omega) d \omega}{\hat{u} \cdot \int_{-\infty}^{\infty} \vec{S}(\vec{r}, \omega) d \omega} .
$$

$\vec{S}(\vec{r}, \omega)$ is the Poynting vector of the Fourier transformed fields $\vec{E}(\vec{r}, \omega)$ and $\vec{H}(\vec{r}, \omega)$ in the pulse. The first term in Eq. (1) is called the net group delay, which is the spectral superposition of group delay at each frequency. The sum of the second and third terms expresses the reshaping delay which is the pulse arrival time at a point without and with the spectral amplitude that is attenuated or amplified during propagation. So the reshaping delay will be zero if the pulse spectrum remains unaltered during propagation. This occurs if the imaginary part of the refractive index does not change with frequency. We have calculated the net group delay using the first term in Eq. (1). The center of mass of the transmitted coherent pulse in our experiments coincides with that in our calculation, with an accuracy of 10 fs. Therefore, the results show quantitatively a good accordance with the prediction by Ref. [13]. However, one can notice a deviation in the propagation time if it is described by a peak of the transmitted pulse. The pulse propagation shown in Fig. 2 may be reminiscent of the Ehrenfest theorem in quantum mechanics. A quantum wave packet propagating in free space may suffer the broadening or distortion owing to the vacuum dispersion while the center of mass of the packet will propagate with a constant velocity of average group velocity $\langle\partial \omega / \partial k\rangle[15]$.

A large reshaping delay may be observed in the propagation of strongly phase modulated chirping pulses in a resonant absorber. By modulating the phase of the coherent pulses, temporally broadened linear down chirping pulses with an autocorrelation width of 335 fs were produced. IR125 dye in dimethyl sulfoxide (DMSO), a resonant absorber with an absorption peak at $794 \mathrm{~nm}$ and a width of $\cong 60 \mathrm{~nm}$ (FWHM), was used as the sample. The effective path length is a product of refractive index and propagation distance $(10 \mathrm{~mm})$, while the refractive index is linearly proportional to the concentration of the dye [16]. Therefore, the propagation distance in our experiment is represented by the concentration of the dye solution. Wavelengths of the incident pulses are tuned at 814 and $774 \mathrm{~nm}$, both sides of the resonance frequency of IR125 absorption band. Figure 3 shows the propagation delays, determined at the center of mass of transmitted pulse, both for coherent and chirping pulses. It can be noted that the propagation delay has been strongly influenced by the chirping factor. On the basis of Eqs. (1) and (2), it is understood that, for a well compressed coherent pulse, the propagation delay is the net group delay. On the other hand, the propagation delay for a chirping pulse includes the effects of both reshaping and net group delays. Therefore, we obtain the reshaping delay experimentally (solid circles in Fig. 3) by subtracting the observed delay of the coherent pulses from that of the chirping pulses. It is interesting to note that the propagation delay for the coherent pulse propagation remain superluminal at both wavelengths as within the anomalous dispersion region. However, for the chirping pulse propagation, it becomes subluminal with an incident wavelength of $814 \mathrm{~nm}$ and more superluminal with that of $774 \mathrm{~nm}$.

Figure 4 displays the results of experiments on transmitted spectra. It shows red and blue spectral shifts with
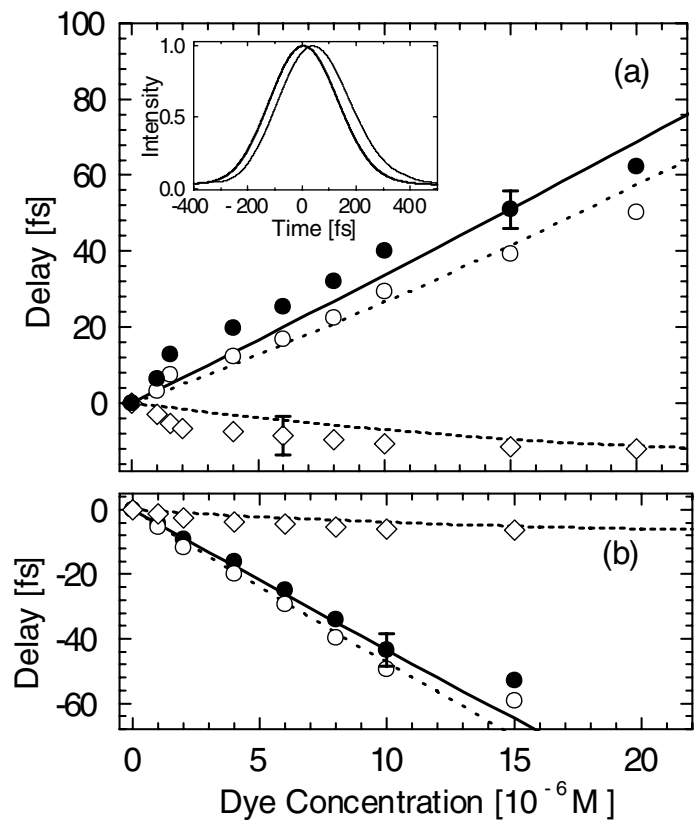

FIG. 3. Observed propagation delays as a function of dye concentration for two different wavelengths (a) 814 and (b) $774 \mathrm{~nm}$. Open circles and open diamonds are propagation delays for chirping and coherent pulses, respectively, while the solid circles represent the measured reshaping delay. Solid, dotted, and dashed lines, respectively, describe the reshaping, total, and net group delays for chirping pulse propagation calculated using Eqs. (1) and (2). Inset displays the temporal profile of a transmitted pulse after propagation through a concentration of the dye of $10 \times 10^{-6} \mathrm{M}$ (thin line) along with the initial (thick line) chirping pulse at $814 \mathrm{~nm}$. 


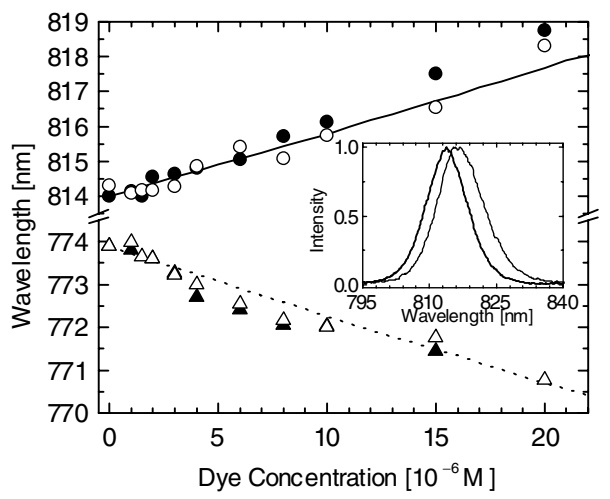

FIG. 4. Solid circles and solid triangles represent the experimentally observed center wavelengths of the transmitted chirping pulses as a function of dye concentration at the incident wavelengths of 814 and $774 \mathrm{~nm}$, respectively, while the open circles and open triangles represent that for coherent pulses. Inset displays spectral profile of the transmitted chirping pulse after propagation through the dye of $10 \times 10^{-6} \mathrm{M}$ (thin line) along with the initial (thick line) chirping pulse at $814 \mathrm{~nm}$.

propagation distance at initial wavelengths of 814 and $774 \mathrm{~nm}$, respectively. The chirping pulse is organized in such a way that the high frequency components are in the front and the low frequency ones are in the back of the pulse in the time domain. In the case of the incident pulse at $774 \mathrm{~nm}$, an enhanced superluminality observed in the propagation delay is attributed to the strong attenuation of the low frequency components of the pulse in the medium. Reversely, a large subluminal effect has been observed for the incident pulse at $814 \mathrm{~nm}$. The observed spectral shifts for chirping and coherent pulses are the same, as the initial pulse spectrum remains unaltered in both cases. It can be noted that such spectral shifts originate in the imaginary part of the refractive index and suggest that the conventional group velocity, described on the basis of the real part of the refractive index only, would not work as well to describe the pulse propagation. The solid lines in Fig. 3 show the reshaping delay calculated using Eq. (1) and the observed spectral shifts. The evaluation order of net group and reshaping delays in Eq. (1) can be interchanged [13]. However, for practical purposes the net group delay at the end of propagation has a greater significance. So, we evaluate the reshaping delay as the difference of propagation time between the transform limited coherent and chirping pulses. The experimental results in Figs. 3 and 4 show quantitatively a good accordance with the prediction by Ref. [13].

Enhanced superluminality observed at $774 \mathrm{~nm}$ for phase modulated chirping pulses may not even be a potential of the superluminal signal propagation. First, one may define the signal position by printing a nonanalytical point in the pulse profile. A nonanalytical point contains nearly an infinite range of frequency components and can seriously deform the transmitted chirping pulse profile. Second, if the signal is defined on the basis of the center of mass, one cannot determine the signal position until the complete pulse has passed the detector. Finally, if one defines the signal when the pulse profile crosses a certain level of the intensity, it is discussed that quantum noise disturbs for such a signal to propagate faster than $c$ [17].

In conclusion, we have measured the propagation delay for different shaped optical pulses in absorbing and absorptionless dispersive media with a good experimental accuracy and described it in the context of the net group and reshaping delays. Increased superluminality or subluminality, observed for phase modulated chirping pulse propagation, is a consequence of the reshaping of output pulses. The propagation of the wave packet can be important in applications, besides its fundamental interests both in classical and quantum physics. A fast communication system requires a broad spectrum. In the dispersion regions, although the profiles suffer a significant deformation, the center of mass of the pulse propagates with the net group delay.

This work was supported by the 21 century COE program "Research and education center of nanovision science." A. I. T. acknowledges support from JSPS.

[1] L. Brillouin, Wave Propagation and Group Velocity (Academic, New York, 1960).

[2] C. G. B. Garret and D. E. McCumber, Phys. Rev. A 1, 305 (1970).

[3] S. Chu and S. Wong, Phys. Rev. Lett. 48, 738 (1982).

[4] A. M. Steinberg, P. G. Kwiat, and R. Y. Chiao, Phys. Rev. Lett. 71, 708 (1993).

[5] D. R. Solli, C. F. McCormick, C. Ropers, J. J. Morehead, R. Y. Chiao, and J. M. Hickmann, Phys. Rev. Lett. 91, 143906 (2003).

[6] A. Ruschhaupt and J.G. Muga, Phys. Rev. Lett. 93, 020403 (2004).

[7] Nicolas Brunner, Valerio Scarani, Mark Wegmuller, Matthieu Legre, and Nicolas Gisin, Phys. Rev. Lett. 93, 203902 (2004).

[8] Lene Vestergaard Hau, S. E. Harris, Zachary Dutton, and Cyrus H. Behroozi, Nature (London) 397, 594 (1999).

[9] C. Liu, Z. Dutton, C. H. Behroozi, and L. V. Hau, Nature (London) 409, 490 (2001).

[10] D. F. Phillips, A. Fleischhauer, A. Mair, R. L. Walsworth, and M. D. Lukin, Phys. Rev. Lett. 86, 783 (2001).

[11] Michael D. Stenner, Daniel J. Gauthier, and Mark A. Neifeld, Nature (London) 425, 695 (2003).

[12] L. J. Wang, A. Kuzmich, and A. Dogariu, Nature (London) 406, 277 (2000).

[13] J. Peatross, S. A. Glasgow, and M. Ware, Phys. Rev. Lett. 84, 2370 (2000).

[14] Marc M. Wefers and Keith A. Nelson, J. Opt. Soc. Am. B 12, 1343 (1995).

[15] Leonard I. Schiff, Quantum Mechanics (McGraw-Hill, New York, 1968), 3rd ed..

[16] Md. Aminul Islam Talukder, Yoshimitsu Amagishi, and Makoto Tomita, Phys. Rev. Lett. 86, 3546 (2001).

[17] A. Kuzmich, A. Dogariu, L. J. Wang, P. W. Milonni, and R. Y. Chiao, Phys. Rev. Lett. 86, 3925 (2001). 